PROCEEDINGS OF THE

AMERICAN MATHEMATICAL SOCIETY

Volume 137, Number 7, July 2009, Pages 2471-2482

S 0002-9939(09)09763-9

Article electronically published on January 6, 2009

\title{
COMBINATORIAL PROOFS OF THE LAMBDA ALGEBRA BASIS AND EHP SEQUENCE
}

\author{
WILLIAM RICHTER \\ (Communicated by Ronald A. Fintushel) \\ AbStract. Combinatorial proofs are given of the $\Lambda$ basis and EHP sequence.
}

\section{INTRODUCTION}

This is the first in a series of papers on geometric applications of Mahowald's Mah67, Mah82 work on the unstable Adams spectral sequence (uAss). The lambda algebra $\Lambda$ (see \$2) was defined by Bousfield et al. $\overline{B C K}+66$, who proved an admissible monomial basis for $\Lambda$, similar to that of the Steenrod algebra $\mathcal{A}$, and constructed a subcomplex $\Lambda(n)$ of $\Lambda$, as the $E_{1}$ term of the uAss for $S^{n}$. Curtis Cur69 claimed a $\Lambda$ EHP sequence (similar to the EHP sequence for spheres Jam57]). These $\Lambda$ results are claimed to have easy combinatorial proofs Cur69, CM89, Koc96, Lin81, HM82, MT94, Rav86. We find this to be a serious pedagogical gap and give combinatorial proofs here. Our proof of the $\Lambda$ basis seems to be the first combinatorial proof, and Bousfield explained it using an action of $\mathcal{A}_{*}$ on $\Lambda$. Our proof of the $\Lambda$ EHP sequence uses $\Lambda$ unstable composition products which are "Adams filtration better" than unstable geometric compositions. The preprint version of Sin75] gave a fine combinatorial treatment of the $\Lambda$ EHP sequence: Singer stated and proved Prop. 1.1, Prop. 5.1, and Thm. 1.2, which he deduced from Prop. 1.1, as we do, but these combinatorial proofs were deleted from the published paper [Sin75].

Our unstable $\Lambda$ composition result [Sin75, Prop. 5.1] implies the $\Lambda$ EHP sequence:

Proposition 1.1 (Singer). Composition in $\Lambda$ restricts to an unstable composition pairing:

$$
\begin{aligned}
\Lambda^{s, t}(n) \otimes \Lambda(n+t) & \rightarrow \Lambda(n), \\
\alpha \otimes \beta & \mapsto \alpha \smile \beta .
\end{aligned}
$$

Theorem 1.2. There is an exact sequence of complexes and a chain map $P$,

$$
\Lambda(n) \stackrel{E}{\longmapsto} \Lambda(n+1) \stackrel{H}{\rightarrow} \Lambda(2 n+1), \quad \Lambda(2 n+1) \stackrel{P}{\longrightarrow} \Lambda(n),
$$

where $H$ and $P$ are defined by $H\left(\lambda_{n} \alpha\right)=\alpha$ and $P(\alpha)=d\left(\lambda_{n}\right) \smile \alpha$, for $\alpha \in$ $\Lambda(2 n+1)$, and $H(\Lambda(n))=0$. $P$ induces the cohomology boundary.

Received by the editors May 2, 2008, and, in revised form, September 12, 2008.

2000 Mathematics Subject Classification. Primary 55T15, 55Q40, 55Q25.

(C)2009 American Mathematical Society 
Proposition 1.1follows by induction from the $s=1$ special case [Mah75, Lem. 3.5] or its "dual" Wan67, Lem. 1.8.1]. Bousfield's explanation of our proof is that the "Curtis-excess" Wan67, Prop. 1.8.2] cannot rise after performing Adem relations. Proposition 1.1 implies the $\Lambda$ EHP sequence, which is not anywhere in the literature both stated and proved (there seems to be no proof following $[\overline{B C K}+66$ ). Wang proved Wan67, Thm. 1.8.4] that $\Lambda(n)$ is a subcomplex of $\Lambda$, and nearly proves Theorem 1.2, but he does not mention $H$ at all.

Bousfield and Kan BK73, 18.1(iv)] construct unstable cohomology compositions (in the $E_{2}$ term of the uAss) compatible with the geometric unstable compositions 1

$$
\begin{aligned}
H^{s, t} \Lambda(n) \otimes H^{*} \Lambda(n+t-s) & \rightarrow H^{*} \Lambda(n), \\
\pi_{n+t-s} S^{n} \otimes \pi_{*} S^{n+t-s} & \rightarrow \pi_{*} S^{n} .
\end{aligned}
$$

Since the differential $d$ of $\Lambda$ preserves the $t$-degree, Proposition 1.1 immediately implies an "Adams-filtration better" improvement of (1.1):

Corollary 1.3 (Singer). Unstable $\Lambda$ composition induces the cohomology composition

$$
H^{s, t} \Lambda(n) \otimes H^{*} \Lambda(n+t) \rightarrow H^{*} \Lambda(n) .
$$

I discovered Prop. 1.1 by comparing the $\Lambda$ EHPss calculations [Rav86, Fig. 3.3.10] with Toda's geometric calculations Tod62. The $E_{2}$ term of the uAss $H^{s, t} \Lambda(n) \Longrightarrow$ $\pi_{n+t-s}\left(S^{n}\right)$ is isomorphic BC70, Thm. 3.3] to $\operatorname{Ext}_{\mathcal{M} \mathcal{A}}^{s}\left(\tilde{H}_{*}\left(S^{n+t}\right), \tilde{H}_{*}\left(S^{n}\right)\right)$, which we write as $\operatorname{Ext}^{s}\left(S^{n+t}, S^{n}\right)$. Under this isomorphism, the Cor. 1.3 composition becomes the Yoneda product in the category $\mathcal{N} \mathcal{A}$ of unstable $\mathcal{A}$-modules Sin75, Prop. 6.6]

$$
Y: \operatorname{Ext}^{s}\left(S^{n+t}, S^{n}\right) \otimes \operatorname{Ext}^{r}\left(S^{z}, S^{n+t}\right) \rightarrow \operatorname{Ext}^{s+r}\left(S^{z}, S^{n}\right) .
$$

Singer explained that he conjectured Prop. 1.1 by expecting a $\Lambda$ product which induces $Y$. Bousfield and Kan's actual $E_{2}$ composition product (1.1) is $Y \circ\left(1 \otimes E^{s}\right)$, using the $s$-fold suspension map $E^{s}: \operatorname{Ext}^{r}\left(S^{z-s}, S^{n+t-s}\right) \rightarrow \operatorname{Ext}^{r}\left(S^{z}, S^{n+t}\right)$ defined in $[\operatorname{Sin} 75, \S 3]$.

Singer's formula [Sin75, Prop. 5.3] for the Hopf invariant of an unstable $\Lambda$ composition is proved in \$5. Mahowald proved a special case Mah75, Prop. 3.1], calculating the suspended Hopf invariant of $P$ (the $\Lambda$ analogue of his conjecture proved in Ric95]). In $\sqrt[6]{6}$ we reprove Wang's result on the equivalence of the admissible and symmetric Adem relations.

\section{The $\Lambda$ ADMISSIBle MONOMIAL BASIS}

Let $V$ be the $\mathbb{Z} / 2$ vector space with basis $\left\{\lambda_{p}: p \geq-1\right\}$. Define $e: V \rightarrow V$ by $e\left(\lambda_{p}\right)=\lambda_{p+1}$, and define the self-map $D=e \otimes 1+1 \otimes e$ of $V^{\otimes 2}$. We'll use the original $[\mathrm{BCK}+66]$ symmetric Adem relations for $p \geq-1, n \geq 0$ :

$$
R(p, 2 p+1+n):=D^{n}\left(\lambda_{p} \otimes \lambda_{2 p+1}\right)=\sum_{i+j=n}\left(\begin{array}{c}
n \\
i
\end{array}\right) \lambda_{p+i} \otimes \lambda_{2 p+1+j} \in V^{\otimes 2} .
$$

\footnotetext{
${ }^{1}$ Actually somewhat less, but Bousfield believes this result can be obtained using [Bou89].
} 
We have a relation $R(p, q) \in V^{\otimes 2}$ for $q>2 p$ and $p \geq-1$. Let $W \subset V$ be the subspace $\mathbb{Z} / 2\left\{\lambda_{p}: p \geq 0\right\}$, and let $I$ be the 2-sided ideal of the tensor algebra $T(W)$ generated by the relations $\{R(p, 2 p+1+n): p, n \geq 0\}$. Let $\Lambda=T(W) / I$ be the resulting quotient algebra. $\Lambda$ is spanned by the monomials $\lambda\left(a_{1}, \ldots, a_{s}\right)=$ $\lambda_{a_{1}} \cdots \lambda_{a_{s}}$, for $a_{i} \geq 0$. Because the relations (and the $d$ formula) are homogeneous, $\Lambda$ is bigraded by $s$ and $t$, where $\lambda\left(a_{1}, \ldots, a_{s}\right)$ has bidegree $s$ and $t=a_{1}+\cdots+a_{s}+s$. We'll write $\Lambda^{s, t}$, and we have $\Lambda=\bigoplus_{s, t \geq 0} \Lambda^{s, t}$. We will speak later of $\Lambda^{s, t}(n)$.

A monomial $\lambda\left(a_{1}, \ldots, a_{s}\right)$ is admissible iff $a_{i} \leq 2 a_{i-1}$ for $1<i \leq s$. We'll often use the right-lexicographical order on monomials and call it the right-lex order. As usual, "performing an Adem relation" means replacing the term $\lambda(p, 2 p+1+n)$ by the other terms that appear in the Adem relation $R(p, 2 p+1+n)$. Performing Adem relations obviously reduces the right-lex order, and each bidegree $(s, t)$ contains only finitely many monomials. Hence, by induction, performing Adem relations, in any order, leads to a sum of admissible monomials. Hence the admissible monomials $\operatorname{span} \Lambda$.

We'll often use the "inner part" of the Adem relations. For $p \geq-1, n>0$, let

$$
\begin{aligned}
\hat{R}(p, 2 p+1+n): & =\lambda(p, 2 p+1+n)+\lambda(p+n, 2 p+1)+R(p, 2 p+1+n) \\
& =\sum_{i+j=n, i j>0}\left(\begin{array}{c}
n \\
i
\end{array}\right) \lambda_{p+i} \otimes \lambda_{2 p+1+j} \in W^{\otimes 2}
\end{aligned}
$$

since only the outer term with $i j=0$ can involve $\lambda_{-1}$. Define the symmetric $d$ formula $[\mathrm{BCK}+66] d\left(\lambda_{n}\right)=\hat{R}(-1, n) \in W^{\otimes 2}$, for $n \geq 0$. Then

$$
d\left(\lambda_{n}\right)=R(-1, n)+\lambda_{-1} \otimes \lambda_{n}+\lambda_{n} \otimes \lambda_{-1}=\sum_{i=1}^{n}\left(\begin{array}{c}
n+1 \\
i
\end{array}\right) \lambda_{i-1} \otimes \lambda_{n-i},
$$

and $d$ extends to a self-map of $T(W)$ satisfying a Leibniz rule $d(\alpha \beta)=d(\alpha) \beta+$ $\alpha d(\beta)$. As we show in $\sqrt{6}, \Lambda$ can be defined by the admissible Adem relations for $p, n \geq 0$ :

$$
\tilde{R}(p, 2 p+1+n):=\lambda_{p} \otimes \lambda_{2 p+1+n}+\sum_{k \geq 0}\left(\begin{array}{c}
n-k-1 \\
k
\end{array}\right) \lambda_{p+n-k} \otimes \lambda_{2 p+1+k} \in V^{\otimes 2} .
$$

In 86 , we also reprove Wang's other result, that the $d$ formula can be defined admissibly as

$$
d\left(\lambda_{n}\right)=\sum_{k>0}\left(\begin{array}{c}
n-k \\
k
\end{array}\right) \lambda_{n-k} \lambda_{k-1} \in \Lambda^{2, n+1}(n) .
$$

We will not use formulas (2.4) and (2.5) in our combinatorial proofs.

We now construct the relations between Adem relations. Define the self-map $C=e \otimes e^{2}$ of $V^{\otimes 2}$ to go with $D=e \otimes 1+1 \otimes e$ defined above. $C$ and $D$ preserve the Adem relations:

$$
C(R(p, q))=R(p+1, q+2), \quad D(R(p, q))=R(p, q+1) .
$$

Call $I=1 \otimes 1$ the identity self-map of $V^{\otimes 2}$. To get relations between Adem relations, we'll define self-maps of $V^{\otimes 3}$ and apply them, for $a \geq-1$, to

$$
\lambda_{a} \otimes \lambda_{2 a+1} \otimes \lambda_{4 a+3}=R(a, 2 a+1) \otimes \lambda_{4 a+3}=\lambda_{a} \otimes R(2 a+1,4 a+3) .
$$


We'll "extend" $C$ and $D$ to $V^{\otimes 3}$ by defining the self-maps $D_{3}=e \otimes I+1 \otimes e \otimes 1+I \otimes e$ and $C_{3}=e \otimes e^{2} \otimes 1+e \otimes 1 \otimes e^{2}+1 \otimes e \otimes e^{2}$ of $V^{\otimes 3}$. Then we can write $C_{3}$ and $D_{3}$ as

$$
\begin{array}{ll}
C_{3}=C \otimes 1+D \otimes e^{2} & D_{3}=D \otimes 1+I \otimes e \\
C_{3}=e \otimes D^{2}+1 \otimes C & D_{3}=e \otimes I+1 \otimes D .
\end{array}
$$

Equations (2.7) express both $C_{3}$ and $D_{3}$ as a sum of two commuting operators on $V^{\otimes 2} \otimes V$. The binomial theorem gives $C_{3}^{n} D_{3}^{m}=\sum_{\substack{i+j=n \\ s+t=m}}\left(\begin{array}{c}n \\ i\end{array}\right)\left(\begin{array}{c}m \\ s\end{array}\right) C^{i} D^{j+s} \otimes e^{2 j+t}$ and

$C_{3}^{n} D_{3}^{m} R(a, 2 a+1) \otimes \lambda_{4 a+3}=\sum_{\substack{i+j=n \\ s+t=m}}\left(\begin{array}{c}n \\ i\end{array}\right)\left(\begin{array}{c}m \\ s\end{array}\right) R(a+i, 2 a+1+n+i+s) \otimes \lambda_{4 a+3+2 j+t}$.

Similarly (2.8) gives $C_{3}^{n} D_{3}^{m}=\sum_{\substack{i+j=n \\ s+t=m}}\left(\begin{array}{c}n \\ i\end{array}\right)\left(\begin{array}{c}m \\ s\end{array}\right) e^{i+s} \otimes D^{2 i+t} C^{j}$ on $V \otimes V^{\otimes 2}$, and

$C_{3}^{n} D_{3}^{m} \lambda_{a} \otimes R(2 a+1,4 a+3)=\sum_{\substack{i+j=n \\ s+t=m}}\left(\begin{array}{c}n \\ i\end{array}\right)\left(\begin{array}{c}m \\ s\end{array}\right) \lambda_{a+i+s} \otimes R(2 a+1+j, 4 a+3+2 n+t)$.

By Equation (2.6), the two displayed right-hand sides are equal, so subtract them to get 0 . But first make the substitutions $b=2 a+1+n$ and $c=2 b+1+m$, for $n, m \geq 0$. Then

$$
\sum_{i+j=n ; s+t=m}\left(\begin{array}{c}
n \\
i
\end{array}\right)\left(\begin{array}{c}
m \\
s
\end{array}\right)\left(\begin{array}{c}
R(a+i, b+i+s) \otimes \lambda_{c-2 i-s} \\
+\lambda_{a+i+s} \otimes R(b-i, c-s)
\end{array}\right)=0 \in V^{\otimes 3} .
$$

These are our relations between Adem relations. Relations (2.9) immediately imply

Lemma 2.1. Given two inadmissible pairs $(a, b)$ and $(b, c)$, with $a \geq-1, b>2 a$, and $c>2 b$, we can rewrite $\lambda_{a} \otimes R(b, c)+R(a, b) \otimes \lambda_{c}$ as a sum

$$
\sum_{i} \lambda_{x_{i}} \otimes R\left(y_{i}, z_{i}\right)+\sum_{j} R\left(e_{j}, f_{j}\right) \otimes \lambda_{g_{j}} \in V^{\otimes 3}
$$

where the triples $\left(x_{i}, y_{i}, z_{i}\right)$ and $\left(e_{j}, f_{j}, g_{j}\right)$ have lower right-lex order than $(a, b, c)$.

We now give our combinatorial proof of the MIT school's result [BCK+66, Pri70].

Theorem 2.2. $\Lambda$ has a basis of the admissible monomials, and $d$ is a well-defined self-map of $\Lambda$ satisfying $d^{2}=0$.

Proof. We'll show more, that $d^{2} \lambda_{c}=0 \in W^{\otimes 3}$, for $c \geq 0$. Take relation (2.9) with $n=0, a=-1$ and $m>0$, so $b=-1$ and $c=m-1$. Note $d\left(\lambda_{c}\right)=\sum_{s=1}^{m-1}\left(\begin{array}{c}m \\ s\end{array}\right) \lambda_{s-1} \otimes$ $\lambda_{c-s}$. Applying the obvious projection $V^{\otimes 3} \rightarrow W^{\otimes 3}$ to relation (2.9), we have

$$
d^{2} \lambda_{c}=\sum_{s=1}^{m-1}\left(\begin{array}{c}
m \\
s
\end{array}\right)\left(d \lambda_{s-1} \otimes \lambda_{c-s}+\lambda_{s-1} \otimes d \lambda_{c-s}\right)=0 \in W^{\otimes 3}
$$

To show $d$ is well defined, we'll show that $d(I) \subset W \otimes I+I \otimes W \subset T(W)$. In relation (2.9), let $a=-1$, and choose $n>0$. Then $b=n-1$ and $c=2 n-1+m$. We will show that $d R(b, c) \in W \otimes I+I \otimes W$. All the terms in (2.9) belong to $W \otimes I+I \otimes W$ except the terms with $i=0$, or $i=n$ and $s=m$, in the first 
summand, and in the second summand, $i=n$ or $i=s=0$. Considering the remaining terms, the sum

$\lambda_{-1} \otimes R(b, c)+R(b, c) \otimes \lambda_{-1}+\sum_{s=0}^{m}\left(\begin{array}{c}m \\ s\end{array}\right)\left(R(-1, b+s) \otimes \lambda_{c-s}+\lambda_{b+s} \otimes R(-1, c-s)\right)$

in $V^{\otimes 3}$ belongs to $W \otimes I+I \otimes W$. Note that $R(b, c)=\sum_{s=0}^{m}\left(\begin{array}{c}m \\ s\end{array}\right) \lambda_{b+s} \otimes \lambda_{c-s}$. The terms containing $\lambda_{-1}$ cancel out, and the resulting equation shows $d$ is well defined:

$$
d R(b, c)=\sum_{s+t=m}\left(\begin{array}{c}
m \\
s
\end{array}\right)\left(d\left(\lambda_{b+s}\right) \otimes \lambda_{c-s}+\lambda_{b+s} \otimes d\left(\lambda_{c-s}\right)\right) \subset W \otimes I+I \otimes W .
$$

$T(W)$ has a basis of the monomials $\mu\left(a_{1}, \ldots, a_{s}\right):=\lambda_{a_{1}} \otimes \cdots \otimes \lambda_{a_{s}}$, which we order by the right-lex order. Writing an element $\alpha \in T(W)$ uniquely as a sum of distinct monomials $\alpha=\sum_{k} \tau_{k}$, we call the leading term of $\alpha$ the term $\tau_{k}$ with maximum right-lex order. This defines a partial order on $T(W)-\{0\}$ : the right-lex order of the leading terms. The 2-sided ideal $I \subset T(W)$ is spanned by the spanning elements, for $s \geq 2, i \geq 1$, and $a_{i+1}>2 a_{i}$ :

$$
S\left(a_{1}, \ldots, a_{s} ; i\right)=\mu\left(a_{1}, \ldots, a_{i-1}\right) \otimes R\left(a_{i}, a_{i+1}\right) \otimes \mu\left(a_{i+2}, \ldots, a_{s}\right) \in T(W),
$$

$S\left(a_{1}, \ldots, a_{s} ; i\right)$ has leading right-lex term $\mu\left(a_{1}, \ldots, a_{s}\right) . T(W)$ has another subspace $A$ with basis the admissible monomials $\mu\left(a_{1}, \ldots, a_{s}\right) \in T(W)$ with $a_{i} \leq 2 a_{i+1}$ for $i<s$. Given $\phi \in I \cap A$, write $\phi$ as a sum of distinct spanning elements $\sigma_{k}$, with $\sigma_{0}=S\left(a_{1}, \ldots, a_{s} ; i\right)$ having maximum order among the $\sigma_{k}$. Since $\phi \in A$, $\mu\left(a_{1}, \ldots, a_{s}\right)$ is the leading term of some other $\sigma_{k}$, say $\sigma_{1}$. We will show that $\sigma_{0}+\sigma_{1}$ is a sum of spanning elements with lower order than $\sigma_{0}$. By induction this will prove that $\phi=0$. So $\sigma_{1}=S\left(a_{1}, \ldots, a_{s} ; f\right)$ for some $f \neq i$, and we can assume $f<i$. If $f+1=i$, we're done by Lemma 2.1, in the case $s=3$, and this illustrates the general case. If $f+1<i$, the proof is illustrated by the case $s=4$, where for some $u, v, x, y \geq-1$, we have

$$
\begin{aligned}
& W^{\otimes 4} \ni \sigma_{0}+\sigma_{1}=R(u, v) \otimes \lambda_{x} \otimes \lambda_{y}+\lambda_{u} \otimes \lambda_{v} \otimes R(x, y) \\
& \quad=R(u, v) \otimes\left(\lambda_{x} \otimes \lambda_{y}+R(x, y)\right)+\left(\lambda_{u} \otimes \lambda_{v}+R(u, v)\right) \otimes R(x, y),
\end{aligned}
$$

and we're done, as performing Adem relations lowers the order. So $I \cap A=\{0\}$.

Remark 2.3. Bousfield rephrased this proof in terms of his unpublished "pension operator" action of $\mathcal{A}_{*}^{\text {op }}$ on $\Lambda$. It's implicit in the MIT school's work $[\mathrm{BCK}+66$, Pri70 that the Adem relations are given by $D=\xi_{1}$ and $C=\xi_{2}$ for $s=2$. The relations between Adem relations (2.9) come from $D_{3}=\xi_{1}$ and $C_{3}=\xi_{2}$ for $s=3$, and formulas (2.8) and (2.7) can be rephrased as the diagonal of $\mathcal{A}_{*}, \Delta\left(\xi_{2}\right)=$ $\xi_{2} \otimes 1+\xi_{1}^{2} \otimes \xi_{1}+1 \otimes \xi_{2}$. By considering action of the higher $\xi_{n}$ on $\Lambda$, we can give an explicit description of Priddy's Koszul free $\mathcal{A}$-module resolution of $\mathbb{Z} / 2$, with which Priddy proved the $\Lambda$ basis. Kochman [Koc96], whose account I found quite helpful while learning $\Lambda$, gives a short false proof (false proof for span, no proof for linear independence) of Theorem 2.2. I believe he could have given a rigorous "geometric" proof, similar to Priddy's Ext proof, as he describes $\Lambda$ in a way similar to [Pri70]. I don't know of a combinatorial proof that $d^{2}=0$ with the admissible (2.5) form of $d$, but that's the only form of $d$ that Ravenel and Kochman give. 


\section{UnstABle LAMBDA ALGEBRA COMPOSITION PRODUCTS}

Define $\Lambda(n) \subset \Lambda$ to be the subspace with basis the admissible monomials $\lambda\left(a_{1}, \ldots, a_{s}\right)$ with $a_{1}<n$. To motivate the proof below, note that the $s=1$ version of Prop. 1.1 is

$$
\lambda_{a} \Lambda(n+a+1) \subset \Lambda(n), \quad \text { for } a<n \text { (proved in [Mah75, Lem. 3.5]), }
$$

which implies that $\lambda_{a} \lambda_{b} \Lambda(n+a+b+2) \subset \Lambda(n)$ for $a<n$ and $b<n+a+1$, and by induction (and considering the identity element), that $\lambda\left(a_{1}, \ldots, a_{s}\right) \in \Lambda(n)$ if the inequalities $a_{i}<n+i-1+\sum_{j<i} a_{j}$ are satisfied, for $i=1, \ldots, s$. Bousfield noted that these inequalities can be restated with the Curtis excess $\kappa$ [Wan67, Prop. 1.8.2], defined by

$$
\kappa\left(\lambda\left(a_{1}, \ldots, a_{s}\right)\right)=\max _{1 \leq i \leq s}\left(a_{i}-(i-1)-\sum_{j<i} a_{j}\right) .
$$

Thus, $\kappa\left(\lambda\left(a_{1}, \ldots, a_{s}\right)\right)<n$ iff $a_{i}<n+i-1+\sum_{j<i} a_{j}$ for $1 \leq i \leq s$. We now prove

Lemma 3.1. If $\alpha=\lambda\left(a_{1}, \ldots, a_{s}\right)$ is admissible, then $\kappa(\alpha)=a_{1}$.

Proof. We'll show the sequence $x_{i}=a_{i}-(i-1)-\sum_{j<i} a_{j}$ is strictly decreasing, and then we'll be done, since $x_{1}=a_{1} \cdot x_{i}-x_{i+1}=2 a_{i}+1-a_{i+1}>0$, by admissibility.

Given monomials $\alpha \in \Lambda^{s, t}$ and $\beta, \kappa(\alpha \beta)=\max \{\kappa(\alpha), \kappa(\beta)-t\}$. This is our product formula, which implies that $\kappa(\alpha \beta)<n$ iff $\kappa(\alpha)<n$ and $\kappa(\beta)<n+t$. Bousfield says the following is Curtis's original proof of [Wan67, Prop. 1.8.2].

Lemma 3.2. For a monomial $\alpha=\lambda\left(a_{1}, \ldots, a_{s}\right)$, if $\kappa(\alpha)<n$, then $\alpha \in \Lambda(n)$.

Proof. If $\alpha$ is admissible, we're done by Lemma 3.1. We'll show that performing an Adem relation on any inadmissible pair in the monomial $\alpha$ writes $\alpha$ as a sum of monomials $\beta_{i}$ with $\kappa\left(\beta_{i}\right)<n$. Then we'll be done, by the proof that the admissibles span. First take $\alpha=\lambda(p, 2 p+1+r)$, so $\kappa(\alpha)=p+r<n$. For each term of $R(p, 2 p+1+r)$ in (2.1), we have $\kappa(\lambda(p+i, 2 p+1+j))=\max \{p+i, p+j-i\} \leq$ $p+r<n$, and we're done. This implies the general case $\alpha=\beta \otimes \lambda(p, 2 p+1+r) \otimes \gamma$ by the product formula.

Singer's result follows immediately from Lemmas 3.1 and 3.2 and our product formula:

Proof of Proposition 1.1. We'll show that $\Lambda^{s, t}(n) \cdot \Lambda(n+t) \subset \Lambda(n)$. Take admissible monomials $\alpha \in \Lambda^{s, t}(n)$ and $\beta \in \Lambda(n+t)$. By Lemma 3.1 $\kappa(\alpha)<n$ and $\kappa(\beta)<n+t$. Thus $\kappa(\alpha \cdot \beta)<n$, by the product formula. Hence $\alpha \cdot \beta \in \Lambda(n)$ by Lemma 3.2 .

The unstable $\Lambda$ composition satisfies an obvious associativity property. If $\alpha \in$ $\Lambda^{s, t}(n), \beta \in \Lambda^{s^{\prime}, t^{\prime}}(n+t)$, and $\gamma \in \Lambda\left(n+t+t^{\prime}\right)$, then

$$
\alpha \smile(\beta \smile \gamma)=(\alpha \smile \beta) \smile \gamma \in \Lambda(n) .
$$

Equality follows from the injection $\Lambda(n) \subset \Lambda$, since the $\Lambda$ composition is associative. 


\section{The lambda algebra EHP SEQUence}

Theorem 2.2 immediately implies a split EHP sequence of vector spaces: the obvious map is an isomorphism $\Lambda(n) \oplus \lambda_{n} \Lambda(2 n+1) \stackrel{\cong}{\longrightarrow} \Lambda(n+1)$, and we have a subcomplex inclusion $E: \Lambda(n) \rightarrow \Lambda(n+1)$. We now prove that $\Lambda(n)$ is a subcomplex [Wan67].

Corollary 4.1. For $n \geq 0$, we have $d \Lambda(n+1) \subset \Lambda(n+1)$.

Proof. We must show that $d\left(\lambda_{n} \alpha\right) \in \Lambda(n+1)$, for any $\alpha \in \Lambda(2 n+1)$. By the Leibniz rule, $d\left(\lambda_{n} \alpha\right)=d\left(\lambda_{n}\right) \alpha+\lambda_{n} d(\alpha)$. We can assume that $d(\alpha) \in \Lambda(2 n+1)$, by induction on $s$, and hence $\lambda_{n} d(\alpha) \in \Lambda(n+1)$. So it suffices to show that $d\left(\lambda_{n}\right) \cdot \Lambda(2 n+1) \subset \Lambda(n+1)$. We'll show one dimension better. By the $d$ formula (2.3) and Proposition 1.1, $d\left(\lambda_{n}\right) \in \Lambda(n)$. Then $d\left(\lambda_{n}\right) \alpha \in \Lambda(n)$ by Proposition 1.1, which says that $\Lambda^{2, n+1}(n) \cdot \Lambda(2 n+1) \subset \Lambda(n)$.

To construct the $\Lambda$ EHP sequence (cf. Cur71]), recall that the Hopf invariant

$$
H: \Lambda(n+1) \rightarrow \Lambda(2 n+1)
$$

is defined so that $H \cdot E=0$, and $H\left(\lambda_{n} \alpha\right)=\alpha$, for $\alpha \in \Lambda(2 n+1)$. Since $E$ is a chain map, we have a quotient complex $\Lambda(n+1) / \Lambda(n)$, but we must show

Corollary 4.2. The linear map $H: \Lambda(n+1) \rightarrow \Lambda(2 n+1)$ is a chain map.

Proof. It suffices to show that $d H=H d$ holds for an element $\lambda_{n} \alpha \in \Lambda(n+1)$, for any $\alpha \in \Lambda(2 n+1)$, since $d \Lambda(n) \subset \Lambda(n)$. Now replicate the proof of Corollary 4.1 .

The [Mah75] description of $P$ is now immediate, and we've proved Theorem 1.2 ,

Remark 4.3. [Koc96, p. 197] incorrectly deduces Cor. 4.2 from the mere fact that $d\left(\lambda_{n}\right) \in \Lambda(n)$. [HM82, pp. 321-322] merely asserts Cor. 4.1, but deduces the easier result that $\Lambda(n)$ is a subring, from the $\Lambda$ version of (1.1), which we will call the $\Lambda$ geometric composition

$$
\Lambda(n) \otimes \Lambda(n+t-s) \rightarrow \Lambda(n),
$$

which Harper and Miller deduce from Mahowald's (3.1). [BC70, Rem. 5.3] constructs a cohomology EHP sequence, but uses Cor.4.2 without proof. Sin75, p. 379] attributes Cor. 4.2 to [BC70, Cur71] (where no proof appears), but [Sin75, §3] uses unstable Ext to construct $H$ in $H^{*} \Lambda$, and in the preprint version of [Sin75], Singer constructs a cohomology EHP sequence in unstable Ext, using a nice argument which he attributes to Bousfield.

\section{The Hopf invariant of AN Unstable $\Lambda$ COMposition}

Recall $S q^{0}$, the algebra homomorphism of $\Lambda$ defined by $S q^{0}\left(\lambda_{a}\right)=\lambda_{2 a+1}$. We'll write $\theta$ for $S q^{0}$ Wan67]. Since $\theta(R(p, 2 p+1+r))=R(2 p+1,4 p+3+2 r)$, $\theta$ is well defined, and $\theta\left(d\left(\lambda_{a}\right)\right)=d\left(\lambda_{2 a+1}\right)$. There's an unstable restriction $\theta: \Lambda^{s, t}(n) \rightarrow$ $\Lambda^{s, 2 t}(2 n)$, and

Proposition 5.1 (Singer). If $\alpha \in \Lambda^{s, t}(n+1)$ and $\beta \in \Lambda(n+t+1)$, then

$$
E H(\alpha \smile \beta)=E H(\alpha) \smile \beta+\theta(\alpha) \smile E H(\beta) \in \Lambda(2 n+2) .
$$


That is, the composite $\Lambda^{s, t}(n+1) \otimes \Lambda(n+t+1) \stackrel{\smile}{\longrightarrow} \Lambda(n+1) \stackrel{E H}{\longrightarrow} \Lambda(2 n+2)$ is the sum of the two composites in the diagram

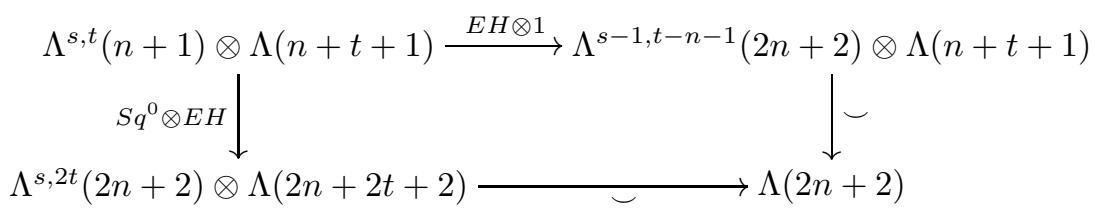

Proof. It suffices to prove the result in $\Lambda$ : if $\alpha \in \Lambda^{s, t}(n+1)$ and $\beta \in \Lambda(n+t+1)$, then

$$
H(\alpha \smile \beta)=H(\alpha) \beta+\theta(\alpha) H(\beta) \in \Lambda .
$$

We'll prove this by induction on $s$. First we'll prove the case $s=1$. So let $\alpha=\lambda_{a}$, with $0 \leq a \leq n$, and write $m=n+a+1$. For $\beta \in \Lambda(m+1)$, we need

$$
H\left(\lambda_{a} \smile \beta\right)=\delta_{a, n} \beta+\lambda_{2 a+1} H(\beta) \in \Lambda .
$$

Assume $a<n$. Write $\beta=\lambda_{m} x+E(y)$, for $x \in \Lambda(2 m+1)$, and $y \in \Lambda(m)$. Using the Adem relation $R(a, m)$ and (2.2), we have $\lambda_{a} \lambda_{m}=\lambda_{n} \lambda_{2 a+1}+\hat{R}(a, m) \in \Lambda$, where $\hat{R}(a, m) \in \Lambda^{2, m+a+2}(n)$. Then $\hat{R}(a, m) \smile x \in \Lambda(n)$, since $n+m+a+2=2 m+1$, by Proposition 1.1. By (3.1), $\lambda_{2 a+1} \smile x \in \Lambda(2 n+1)$ and $\lambda_{a} \smile y \in \Lambda(n)$. Then we have

$$
\lambda_{a} \smile \beta=\lambda_{n}\left(\lambda_{2 a+1} \smile x\right)+E\left(\hat{R}(a, m) \smile x+\lambda_{a} \smile y\right) \in \Lambda(n+1),
$$

so $H\left(\lambda_{a} \smile \beta\right)=\lambda_{2 a+1} H(\beta) \in \Lambda$. This finishes the case $a<n$. For $a=n$, write $\beta \in \Lambda(2 n+2)$ in admissible form as $\beta=\lambda_{2 n+1} H(\beta)+E(y)$, for $y \in \Lambda(2 n+1)$. Since $\lambda_{n} \lambda_{2 n+1}=0$, we have $\lambda_{n} \smile \beta=\lambda_{n} y$, and the $s=1$ case (5.3) is concluded by

$$
H\left(\lambda_{n} \smile \beta\right)=y=\beta+\lambda_{2 n+1} H(\beta) \in \Lambda .
$$

The induction step with $s>1$ follows from the strict associativity of the RHS. Take

$$
\alpha \otimes \beta \otimes \gamma \in \Lambda^{s, t}(n+1) \otimes \Lambda^{s^{\prime}, t^{\prime}}(n+t+1) \otimes \Lambda\left(n+t+t^{\prime}+1\right) .
$$

Assuming (5.2) for $s, s^{\prime} \geq 1$, we'll show it's true for $s+s^{\prime}$. Using (3.3), we have

$$
\begin{aligned}
& H((\alpha \smile \beta) \smile \gamma)=H(\alpha \smile(\beta \smile \gamma))=H(\alpha)(\beta \smile \gamma)+\theta(\alpha) H(\beta \smile \gamma) \\
= & H(\alpha) \beta \gamma+\theta(\alpha)(H(\beta) \gamma+\theta(\beta) H(\gamma)) \\
= & (H(\alpha) \beta+\theta(\alpha) H(\beta)) \gamma+\theta(\alpha) \theta(\beta) H(\gamma)=H(\alpha \smile \beta) \gamma+\theta(\alpha \smile \beta) H(\gamma) \in \Lambda .
\end{aligned}
$$

So (5.2) is true with $\alpha \smile \beta$ in the first argument. But every $\alpha \in \Lambda^{s, t}(n+1)$ is a sum of such products: write $\alpha$ admissibly as $\alpha=\sum_{i=0}^{n} \lambda_{i} \smile E\left(x_{i}\right)$, for $x_{i} \in$ $\Lambda^{s-1, t-i-1}(2 i+1)$.

There are two important special cases when Proposition 5.1 desuspends. First, when the second argument $\beta$ desuspends, we have [Sin75, Prop. $5.2 \&$ Prop. 3.7]

Corollary 5.2 (Singer). For $\alpha \in \Lambda^{s, t}(n+1)$ and $\beta \in \Lambda(n+t)$, we have

$$
H(\alpha \smile E(\beta))=H(\alpha) \smile \beta \in \Lambda(2 n+1) .
$$

This follows directly from Prop. 1.1? if we write $\alpha=\lambda_{n} x+E(y)$ admissibly, then $\alpha \beta=\lambda_{n}(x \smile \beta)+E(y \smile \beta)$ is also written admissibly. We also have a result when the first argument desuspends [Sin75, Prop. 6.7] (see [Hik04, Cor. 2.9] for a direct proof): 
Corollary 5.3 (Singer). For $\alpha \in \Lambda^{s, t}(n)$ and $\beta \in \Lambda(n+t+1)$, we have

$$
H(E(\alpha) \smile \beta)=E(\theta(\alpha)) \smile H(\beta) \in \Lambda(2 n+1) .
$$

To motivate Prop. 5.1, recall the two-term formula [BS68, Thm. 3.16] for the suspended Hopf invariant of a composition (cf. Ric97, Thm. 2.7]). With the $\Lambda$ geometric composition (4.1), Cor. 5.2 shows that $H(\alpha \smile \beta)=H(\alpha) \smile \beta$. This corresponds to one of the BS68 two terms, and Mahowald says the other term vanishes in $\Lambda$ due to the higher Adams filtration. With unstable $\Lambda$ composition, we get a different second term, involving $\theta=S q^{0}$.

There are $\Lambda$ analogues (cf. [Sin75, p. 382] of the geometric EHP constructions of Toda, Barratt and others. More calculations will appear in a sequel, but consider Toda's calculation Tod62 of $\pi_{7}^{s}=\mathbb{Z} / 16$, generated by $\sigma \in \pi_{15} S^{8}$, involving $\sigma^{\prime}$, $\sigma^{\prime \prime}$ and $\sigma^{\prime \prime \prime}$, which are born on $S^{7}, S^{6}$ and $S^{5}$ and are stably $2 \sigma, 4 \sigma$ and $8 \sigma$, with Hopf invariants $\eta, \eta^{2}$ and $\eta^{3}$ respectively. $\sigma^{\prime}$ and $\sigma^{\prime \prime}$ are hard to construct, but the $\Lambda$ analogue is easy. Starting with the cycle $\lambda_{7} \in \Lambda(8)$, with $H\left(\lambda_{7}\right)=* \epsilon$ $\Lambda$ (15), Prop. 1.1 and Cor. 5.3 imply $\lambda_{0} \lambda_{7} \in \Lambda(7), \lambda_{0}^{2} \lambda_{7} \in \Lambda(6), \lambda_{0}^{3} \lambda_{7} \in \Lambda(5)$, with $H\left(\lambda_{0} \lambda_{7}\right)=\lambda_{1}, H\left(\lambda_{0}^{2} \lambda_{7}\right)=\lambda_{1}^{2}, H\left(\lambda_{0}^{3} \lambda_{7}\right)=\lambda_{1}^{3}$. Note that $\lambda_{0}^{3} \lambda_{7}$ is therefore a cycle with leading term 4111. Compare [Rav86, Ex. 3.3.11], where 4111 is completed to a cycle by a Curtis algorithm calculation.

Taking $\alpha=d \lambda_{n} \in \Lambda^{2, n+1}(n)$, Prop. 1.1 and (2.3) imply that $H(\alpha)=(n-1) \lambda_{0}$. Prop. 5.1 and Theorem 1.2 immediately imply [Mah82, Prop. 3.1]: The composition

$$
\Lambda(2 n+1) \stackrel{P}{\longrightarrow} \Lambda(n) \stackrel{H}{\longrightarrow} \Lambda(2 n-1) \stackrel{E}{\longrightarrow} \Lambda(2 n)
$$

sends $\beta$ to $(n-1) \lambda_{0} \smile \beta+\theta\left(d \lambda_{n}\right) \smile H(\beta)$. Then $d \lambda_{2 n+1}=E \theta\left(d \lambda_{n}\right)$, and specializing to $n$ even, Mahowald observed that the composition

$$
\Lambda(4 n+1) \stackrel{P}{\longrightarrow} \Lambda(2 n) \stackrel{H}{\longrightarrow} \Lambda(4 n-1) \stackrel{E^{2}}{\longrightarrow} \Lambda(4 n+1)
$$

sends $\beta$ to $\lambda_{0} \smile \beta+d\left(\lambda_{4 n+1}\right) \smile H(\beta)$. Recall the Hilton-Hopf expansion BS68:

$$
2 \iota \cdot \alpha=\alpha \cdot 2 \iota+\left[\iota_{n}, \iota_{n}\right] \cdot H(\alpha) \text {, for } \alpha \in \pi_{*}\left(S^{n}\right) .
$$

It is well-known that $d\left(\lambda_{n}\right)$ corresponds to $\left[\iota_{n}, \iota_{n}\right]$ and $\lambda_{0}$ corresponds to $2 \iota$. Assuming this, we find that (1.1) leads us to expect that left/right composition with $\lambda_{0}$ corresponds to left/right geometric composition by $2 \iota$. Mahowald then observed the following result:

Proposition 5.4 (Mahowald). The composition

$$
\Lambda(4 n+1) \stackrel{P}{\longrightarrow} \Lambda(2 n) \stackrel{H}{\longrightarrow} \Lambda(4 n-1) \stackrel{E^{2}}{\longrightarrow} \Lambda(4 n+1)
$$

induces a self-map of $H^{*} \Lambda(4 n+1)$, which is $E^{2} \cdot H \cdot P(\beta)=\beta \lambda_{0}$.

Proof. We only need to prove the $\Lambda$ analogue of Equation (5.4). We'll prove this directly, but it follows from a general result [Sin75, Thm. 4.1], which is the $\Lambda$ analogue of the Barratt-Toda commutation formula Tod62. For a cycle $f \in$ $\Lambda(p+1)$, we will show

$$
\lambda_{0} \smile f+f \smile \lambda_{0}=d\left(\lambda_{p+1}\right) \smile H(f) \in H^{*} \Lambda(p+1) .
$$

To prove this, write $f$ admissibly as $f=\lambda_{p} A+B$, for $B \in \Lambda(p)$ and $A \in \Lambda(2 p+1)$. Since $f$ is a cycle, $A$ must be a cycle, since $d$ is a chain map, by Corollary 4.2 , 
By definition, commutation with $\lambda_{-1}$ in $T(V)$ is essentially the boundary map $d$, so $0=d f=\left[f, \lambda_{-1}\right] \in T(V)$. Now we'll extend our operator $D$ to $T(V)$, so $D$ satisfies the Leibniz rule, and $D\left(\lambda_{p}\right)=\lambda_{p+1}$. Writing $D(\alpha)=\alpha^{\prime}$, we have

$$
0=\left[f, \lambda_{-1}\right]^{\prime}=\left[f^{\prime}, \lambda_{-1}\right]+\left[f, \lambda_{0}\right]=d\left(f^{\prime}\right)+\left[f, \lambda_{0}\right] \in \Lambda,
$$

so $d\left(f^{\prime}\right)=\left[f, \lambda_{0}\right]=\lambda_{0} f+f \lambda_{0} \in \Lambda$. To make that argument rigorous, note that $d\left(\lambda_{n}\right)=\widehat{R}(-1, n) \in T(W)$, and $d\left(\lambda_{n}\right)=R(-1, n)+\left[\lambda_{n}, \lambda_{-1}\right] \in T(V)$. Using this, the Leibniz rule, and induction, we can then show that $d(g)^{\prime}=d\left(g^{\prime}\right)+\left[g, \lambda_{0}\right] \in$ $T(W)$, for any $g \in T(W)$. We need to show that $d\left(f^{\prime}\right)$ is cohomologous to $d\left(\lambda_{p+1}\right) \smile H(f) \in \Lambda(p+1)$. First note that $\Lambda(k)^{\prime} \subset \Lambda(k+1)$, because for a monomial $C=\lambda\left(a_{1}, \ldots, a_{s}\right) \in \Lambda(k), \kappa\left(C^{\prime}\right) \leq \kappa(C)+1$. So

$$
\begin{aligned}
f^{\prime} & =\lambda_{p+1} A+\lambda_{p} A^{\prime}+B^{\prime}, \\
\lambda_{0} f+f \lambda_{0}=d\left(f^{\prime}\right) & =d\left(\lambda_{p+1}\right) A+d\left(\lambda_{p} A^{\prime}+B^{\prime}\right) \in \Lambda,
\end{aligned}
$$

since $d(A)=0$. But $\left(\lambda_{p} A^{\prime}+B^{\prime}\right) \in \Lambda(p+1)$ by $\Lambda(k)^{\prime} \subset \Lambda(k+1)$ and (3.1). Since $H(f)=A$, we've proved our formula (5.5) .

Ric95 proved that $\Omega^{3} S^{4 n+1} \stackrel{\Omega(P)}{\longrightarrow} \Omega S^{2 n} \stackrel{H}{\longrightarrow} \Omega S^{4 n-1} \stackrel{E^{2}}{\longrightarrow} \Omega^{3} S^{4 n+1}$ is homotopic to the $H$-space squaring map on $\Omega^{3} S^{4 n+1}$, which Mahowald conjectured based on Proposition 5.4. This result implies the following infinite statement in homotopy groups Ric95]:

$$
2 \pi_{k} S^{4 n+1} \subset E^{2}\left(\pi_{k-2} S^{4 n-1}\right), \quad \text { for } k \geq 3 .
$$

The argument of $B C G+95$ strongly indicates that (5.7) can't be deduced from Jam57, Sel84, even though (5.7) does not improve on the James-Selick 2-primary exponent.

\section{Symmetric And ADmissible Adem Relations}

We reprove Wang's result [Wan67, Thm. 1.6.1] (cf. [Koc96]) that the admissible Adem relations (2.4) are equivalent to the symmetric Adem relations (2.1), using a simple recursion formula due to Tangora [Tan78. Define $C_{n, k} \in \mathbb{Z} / 2$, for $n \geq$ $0, k \in \mathbb{Z}$ by

$$
C_{0, k}=0, \quad C_{1, k}=\delta_{k, 0}, \text { and, for } n \geq 2, \quad C_{n, k}=C_{n-1, k}+C_{n-2, k-1} .
$$

Then for $p \geq-1$, and $n \geq 0$, we define

$$
S(p, 2 p+1+n):=\lambda_{p} \otimes \lambda_{2 p+1+n}+\sum_{k} C_{n, k} \lambda_{p+n-k} \otimes \lambda_{2 p+1+k} \in V^{\otimes 2} .
$$

By induction on $n, S(p, 2 p+1+n)$ is a sum of admissibles: $C_{n, k}=0$ for $k<0$ or $2 k+1>n$, and furthermore, $C_{n, 0}=1$ for $n \geq 0$. We now prove

Lemma 6.1. For all $p \geq-1$ and $n \geq 0, S(p, 2 p+1+n)$ equals $\tilde{R}(p, 2 p+1+n)$ of (2.4).

Proof. We will show that $C_{n, k}=\left(\begin{array}{c}n-k-1 \\ k\end{array}\right)$, for $k \geq 0$ and $2 k+1 \leq n$ by induction, the Tangora recursion formula (6.1), and Pascal's triangle:

$$
C_{n+1, k}=C_{n, k}+C_{n-1, k-1}=\left(\begin{array}{c}
n-k-1 \\
k
\end{array}\right)+\left(\begin{array}{c}
n-k-1 \\
k-1
\end{array}\right)=\left(\begin{array}{c}
n-k \\
k
\end{array}\right) .
$$

Now we relate the symmetric and admissible Adem relations by the procedure, which Mahowald stresses, of applying $D$ to formula (6.2): 
Lemma 6.2. For $p \geq-1, S(p, 2 p+1+n)=R(p, 2 p+1+n)$ for $n=0,1,2$, and (6.3) $S(p, 2 p+1+n+1)=D S(p, 2 p+1+n)+S(p+1,2 p+1+n) \in V^{\otimes 2}, \quad$ for $n \geq 2$.

Proof. The first statement is obvious, and (6.3) follows, after examining the coefficients of $\lambda_{p+n+1-k} \otimes \lambda_{2 p+1+k}$, from the equation $C_{n+1, k}=C_{n, k}+C_{n, k-1}+C_{n-2, k-2}$, and this follows from applying the Tangora recursion formula (6.1) twice.

By Lemma 6.2 and induction and $D R(p, q)=R(p, q+1)$, we can write the $S$ relations as sums of the $R$ relations and also write the $R$ relations as sums of the $S$ relations. For $p=-1$, Lemma 6.2 and induction enable us to write $S(-1, q)$ as $R(-1, q)$ plus "positive" Adem relations, and similar to $d\left(\lambda_{n}\right)=\hat{R}(-1, n)$, the admissible formula (2.5) for $d\left(\lambda_{n}\right) \in W^{\otimes 2}$ equals $S(-1, n)+\lambda_{-1} \otimes \lambda_{n}+\lambda_{n} \otimes \lambda_{-1} \in$ $V^{\otimes 2}$. Hence

Lemma 6.3. $\Lambda$ can be defined by either the admissible (2.4) or the symmetric (2.1) Adem relations, and the admissible (2.5) formula for $d$ holds.

For hand calculations, the formulas (6.1) are very convenient. For the $\lambda_{p}$ "page", start with the two rows $\lambda_{p} \lambda_{2 p+1}=0$ and $\lambda_{p} \lambda_{2 p+2}=\lambda_{p+1} \lambda_{2 p+1}$. To make the row with LHS $\lambda_{p} \lambda_{2 p+1+n}$, push the previous row RHS by the vector $(0,-1)$ and the RHS two rows back by $(1,-2)$.

\section{Acknowledgments}

Thanks to Mark Mahowald for explaining his uAss applications to 3-cell Poincaré complexes and the embedding dimension of $\eta_{j}$ as an exotic sphere and for advising me (cf. [Mah82, p. 78]) on my proof of the $\Lambda$ basis. Thanks also to Paul Goerss for many tutorials on the uAss, to Pete Bousfield for many interesting discussions, and to Bill Singer for many discussions about [Sin75]. Thanks also to Mizuho Hikida for discussing [Hik04, which has nice applications of the $\Lambda$ EHP sequence. Finally, thanks to Bob Bruner, John Klein, John Palmieri, Stewart Priddy, Charles Rezk, Marty Tangora, and the referee.

\section{REFERENCES}

[BC70] A. K. Bousfield and E. B. Curtis, A spectral sequence for the homotopy of nice spaces, Trans. Amer. Math. Soc. 151 (1970), 457-479. MR0267586 (42:2488)

[BCG+95] M. G. Barratt, F. Cohen, B. Gray, M. Mahowald, and W. Richter, Two results on the 2-local EHP spectral sequence, Proc. Amer. Math. Soc. 123 (1995), 1257-1261. MR1246514 (95e:55018)

[BCK+66] A. K. Bousfield, E. B. Curtis, D. M. Kan, D. G. Quillen, D. L. Rector, and J. W. Schlesinger, The mod-p lower central series and the Adams spectral sequence, Topology 5 (1966), 331-342. MR0199862 (33:8002)

[BK73] A. K. Bousfield and D. M. Kan, Pairings and products in the homotopy spectral sequence, Trans. Amer. Math. Soc. 177 (1973), 319-343. MR0372860 (51:9064)

[Bou89] A. K. Bousfield, Homotopy spectral sequences and obstructions, Israel J. Math. 66 (1989), 54-104. MR.1017155 (91a:55027)

[BS68] J. M. Boardman and B. Steer, On Hopf invariants, Comment. Math. Helv. 42 (1968), 217-224. MR0221503 (36:4555)

[CM89] E. Curtis and M. Mahowald, The unstable Adams spectral sequence for $S^{3}$, Algebraic Topology (Evanston, IL, 1988), Contemp. Math., vol. 96, Amer. Math. Soc., 1989, pp. 125-162. MR:1022678 (91b:55012)

[Cur69] E. Curtis, Some nonzero homotopy groups of spheres, Bull. Amer. Math. Soc. 75 (1969), 541-544. MR0245007(39:6320) 
[Cur71] , Simplicial homotopy theory, Advances in Math. 6 (1971), 107-209. MR0279808 (43:5529)

[Hik04] M. Hikida, Some acyclic relations in the lambda algebra, Hiroshima Math. J., 34 (2004), 147-160. MR2086839 (2005f:55016)

[HM82] J. Harper and H. Miller, On the double suspension homomorphism at odd primes, Trans. Amer. Math. Soc. 273 (1982), 319-331. MR664045 (83h:55037)

[Jam57] I. M. James, On the suspension sequence, Ann. of Math. (2) 65 (1957), 74-107. MR0083124(18:662e)

[Koc96] S. O. Kochman, Bordism, stable homotopy and Adams spectral sequences, Fields Institute Monographs, vol. 7, Amer. Math. Soc., 1996. MR.1407034 (97i:55017)

[Lin81] W. H. Lin, Algebraic Kahn-Priddy theorem, Pacific J. Math. 96 (1981), 435-455. MR.637982 (83c:55025)

[Mah67] M. Mahowald, On the metastable homotopy of $S^{n}$, Memoirs of the Amer. Math. Soc. 72 (1967), 1-81. MR0236923 (38:5216)

[Mah75] _ On the double suspension, Trans. Amer. Math. Soc. 214 (1975), 169-178. MR0438333 (55:11248)

[Mah82] , The image of J in the EHP sequence, Ann. of Math. (2) 116 (1982), 65-112. MR662118 (83i:55019)

[MT94] M. Mahowald and R. Thompson, The fiber of the secondary suspension map, Amer. J. Math. 116 (1994), 179-205. MR.1262430 (95f:55012)

[Pri70] S. Priddy, Koszul resolutions, Trans. Amer. Math. Soc. 152 (1970), 39-60. MR0265437 $(42: 346)$

[Rav86] D. Ravenel, Complex cobordism and stable homotopy groups of spheres, Pure and Applied Math., vol. 121, Academic Press, 1986. MR860042 (87j:55003)

[Ric95] W. Richter, The H-space squaring map on $\Omega^{3} S^{4 n+1}$ factors through the double suspension, Proc. Amer. Math. Soc. 123 (1995), 3889-3900. MR.1273520 (96b:55014)

[Ric97] A homotopy theoretic proof of Williams's Poincaré embedding theorem, Duke Math. J. 88 (1997), 435-447. MR1455528 (98e:57046)

[Sel84] P. Selick, 2-primary exponents for the homotopy groups of spheres, Topology 23 (1984), 97-99. MR721456 (85k:55011)

[Sin75] W. Singer, The algebraic EHP sequence, Trans. Amer. Math. Soc. 201 (1975), 367382. MR0385861 (52:6720)

[Tan78] M. Tangora, Generating Curtis tables, Algebraic Topology (Proc. Conf., Univ. British Columbia, Vancouver, B.C.), Lecture Notes in Math., vol. 673, Springer, 1978, pp. 243253. MR.517096 (80d:55023)

[Tod62] H. Toda, Composition methods in the homotopy groups of spheres, Princeton Univ. Press, 1962. MR0143217 (26:777)

[Wan67] J. Wang, On the cohomology of the mod-2 Steenrod algebra and the non-existence of elements of Hopf invariant one, Illinois J. Math. 11 (1967), 480-490. MR0214065 $(35: 4917)$

Department of Mathematics, Northwestern University, Evanston, Illinois 60208 